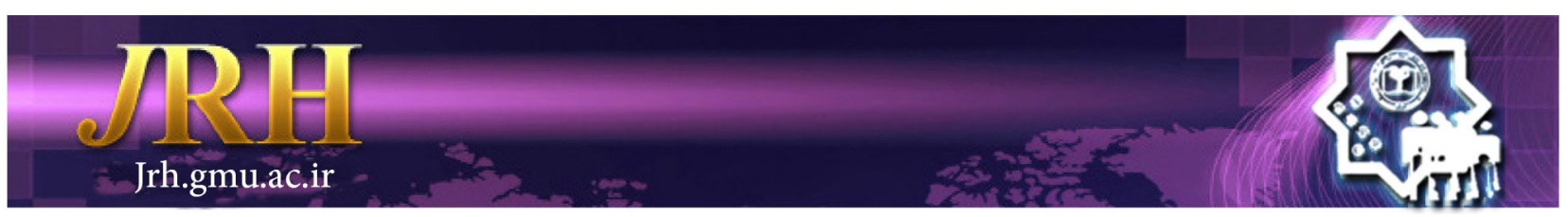

\title{
Effects of aromatherapy with lavender essential oil on sleep quality among retired older adults
}

Fatemeh Sadat Izadi-Avanji ${ }^{1}$, Sedigheh Miranzadeh ${ }^{1}$, Hossein Akbari² Neda Mirbagher Ajorpaz ${ }^{3}$, Darius Ahmadi ${ }^{1}$

\author{
Journal of Research \& Health \\ Social Development \& Health Promotion \\ Research Center \\ Vol. 9, No. 5, Sep \& Oct 2019 \\ Pages: $437-442$ \\ DOI: $10.29252 / j r h .9 .5 .437$ \\ Original Article
}

\begin{abstract}
1. Department of Medical Surgical Nursing, Faculty of Nursing and Midwifery, Trauma Nursing Center, Kashan University of Medical Sciences, Kashan, Iran

2. Department of Biostatistics and Public Health, Faculty of Health, Kashan University of Medical Sciences, Kashan, Iran

3. Department of Operating Room, School Nursing and Midwifery, Autoimmune Diseases Research Center, Kashan University of Medical Sciences, Kashan, Iran

Correspondence to: Fatemeh Sadat IzadiAvanji, Department of Medical Surgical Nursing, Faculty of Nursing and Midwifery, Trauma Nursing Center, Kashan University of Medical Sciences, Kashan, Iran.

Email: Fs.izadi@gmail.com
\end{abstract}

Received: 11 Dec 2016

Accepted: 11 Jun 2018

How to cite this article: Izadi-Avanji FS, Miranzadeh S, Akbari H, Mirbagher Ajorpaz N. Effects of aromatherapy with lavender essential oil on sleep quality among retired older adults. J Research Health2019; 9(5): 437- 442

\section{Introduction}

Poor sleep quality is one of the most common problems among older adults. With aging, some changes occur in the circadian rhythm of sleep along with subsequent reductions in sleep quality. Beside physiological factors, psychological factors such as depression and anxiety, environmental factors, pain, and physical discomfort have roles in the sleep quality in older adults [1]. A study by Tardy et al. showed that about $42 \%$ of older adults have difficulties in the beginning or maintaining sleep [2]. Older adults spend more time in bed than younger people do, but they have less duration of sleep. Based on studies conducted in Iran, sleep problems affect $67 \%$ of older adults [3]. Research has shown that poor sleep quality is the third largest problem after headaches and digestive disorders in older adults [2]. If sleep disorder continues for a long time, it can lead to a chain of unpleasant consequences such as decreased cognitive performance, increased risk of falls, daytime fatigue, mood disorder, decreased immune function, cardiovascular diseases and ultimately, increased risk of 
mortality [4,5]. According to Tardy et al, the use of benzodiazepines and barbiturates will increase insomnia up to $50 \%$ and can lead to poor sleep quality, drug dependency, and increased tolerance and recurrence of insomnia [2]. Given the problems of insomnia as well as side effects of the prescribed sedative drugs, it seems absolutely necessary to start nonpharmacological treatment for the control of insomnia in older adults [6]. Research on palliative, safe, and non-pharmacological interventions with minimal side effects is possible. Non-pharmacological interventions have recently been considered as a better firstline treatment for sleep problems in older adults [7]. Aromatherapy has been used widely for modification of mood disorders and sleep problems.

A research showed that the scent of lavender and chamomile makes the mind of people ready for sleep [8]. Main ingredients of lavender essential oil include Linaly acetate and Linalool. Sedative properties of lavender essential oil have been mentioned in the relevant literature [9]. Reports indicate that lavender essential oil may induce sedative effects by affecting GABAA receptors [10]. Lewith et al. and Goel et al. showed that aromatherapy with lavender essential oil improved sleep quality in people suffering from insomnia [11,12]. However, other studies have not reported the effects of lavender essential oil on sleep quality $[13,14]$. The available studies do not provide sufficient evidence to prove the hypnotic or sedative properties of lavender essential oil [15-18]. As there is no study conducted on the effect of inhalation of this scent on the sleep quality in older adults, as well as due to the preference of drug interactions to relieve sleep problems, this study was conducted to examine the effects of aromatherapy with lavender essential oil on sleep quality in retired older adults.

\section{Method}

This randomized, single-blind, controlled clinical trial was conducted on 100 older adults in 2017. In this study, the participants were unaware of the type of intervention given to every participant (single blinding). The sample size was calculated based on data obtained from a study by Moeini et al. [18]. The minimum sample sized with confidence level of $0.95 \%$ and power of $90 \%$ was determined as 10 persons for each group. The researcher referred to the research setting (educational retirement center in kashan city, Southeast of iran) and used nonprobability-convenience sampling to evaluate all older adults for inclusion criteria. The retirement center had 240 members among whom 130 met the inclusion criteria. 21 persons refused to participate in the study. Finally, 109 participants were selected for the study and randomly were assigned into two groups. 9 participants refused to continue the participation in study. Therefore, data analysis was performed on 100 participants. In the next step, written informed consent was obtained and a socio-demographic questionnaire and Pittsburgh Sleep Quality Index were completed by the participants.

After obtaining permission from the Ethics committee of Kashan university of medical sciences and registration of the present study in the Iranian registry of clinical trials (code: IRCT2013062013722N1), the study wasstarted. Participants who met the inclusion criteria were provided with sufficient information about the procedure. Writteninformed consent was obtained from all participants who had announced their cooperation.

The inclusion criteria were age of 60 or above, being able to communicate either by speaking or by writing, no history of asthma, and no olfactory disorders. In addition, older adults who obtained a score above 5 from the Pittsburgh questionnaire were included in the study.

Older adults with no history of mental disorders, no use of herbal remedies, no use of alternative medicine in the past one week, and those who obtained a score of 24 or above from the questionnaire of Mini Mental State Examination (MMSE) were included in the study. The MMSE is a reliable and easy questionnaire to evaluate cognitive function in 
older adults. The exclusion criteria were having allergy to flowers and plants and having olfactory disorder.

The Pittsburgh Sleep Quality Index (PSQI) was used to examine sleep quality. PSQI is a selfrated instrument frequently used to measure the quality and patterns of sleep in older adults. The total index score is between 0 and 21. A total score of 5 or greater indicates a "poor" quality of sleep [16]. Reliability of PSQI in a study in older adults was calculated using Cronbach's alpha internal consistency coefficient that was reported as 0.80 [17]. In the present study, the reliability of tools was reviewed using Cronbach's alpha and its value was calculated as 0.87 .

The lavender essential oil was used in the intervention group and distilled water as placebo in the control group. Prior to going to bed, the participants poured two drops of the lavender or distilled water on a piece of cotton and then put it under the outer cover of the pillow [18]. The intervention was carried out for 7 nights. The participants were informed that the sleep environment should be quiet with lowest noise and light. Lavender essential oil and placebo were prepared by Barij Essence Pharmaceutical Company in Kashan. Bottles of distilled water and lavender essential oil were completely similar in appearance (odor, color, and shape). To ensure that whether or not participants used lavender essential oil and placebo, they were followed up by daily calls. Seven nights after the intervention, all participants filled out the sleep quality questionnaire again. The day before the participants were to complete the questionnaire, a phone call was made to remind them.

The data were analyzed using SPSS-16. The normality of the quantitative data was evaluated using Kolmogorov-Smirnov test. Sleep quality score was normal before and after the intervention. Independent t-test, Chi-square, and Fisher's exact test were used to study the homology of the study groups. Independent t-test was used to compare the mean scores of quality of sleep before the intervention between the groups. The level of significance was considered below 0.05 at all tests.

\section{Results}

Socio-demographic characteristics of the participants in the intervention and control groups are provided in Table 1 . The results showed that there were no statistically significant differences between the two groups regarding age, gender, marital status, education,

Table 1 Socio-demographic characteristics of the participants in the intervention and control groups

\begin{tabular}{lccc}
\hline Variable & $\begin{array}{c}\text { Lavender Essential oil }(\mathrm{n}=50) \\
\mathrm{n}(\%)\end{array}$ & $\begin{array}{c}\text { Placebo }(\mathrm{n}=50) \\
\mathrm{n}(\%)\end{array}$ & p-value \\
\hline Gender & & & \\
\hline Male & $32(64)$ & $33(66)$ & $0.86^{*}$ \\
Female & $18(36)$ & $17(34)$ & \\
\hline Marital status & & $48(96)$ & \multirow{2}{*}{$0.72^{*}$} \\
\hline Married & $46(92)$ & $2(4)$ & \\
Alone (widowed, divorced, single) & $4(8)$ & & \\
\hline Education & & $4(8)$ & \\
\hline Illiterate & $6(12)$ & $24(36)$ & $0.11^{* *}$ \\
Elementary education & $23(46)$ & $4(4)$ & \\
Diploma & $15(30)$ & & \\
Academic education & $6(6)$ & $22(44)$ & $0.4^{*}$ \\
\hline Intake of hypnotic drugs & & $28(56)$ & \\
\hline Yes & $23(46)$ & Mean $\pm \mathrm{SD}$ & $0.13^{* * *}$ \\
No & $27(54)$ & $68.34 \pm 5.09$ & \\
Age & Mean $\pm \mathrm{SD}$ & & \\
\hline
\end{tabular}

*Fisher exact test

$* *$ Chi-squared test

$* * *$ Independent $\mathrm{t}$ test 
and history of taking sleeping pills and the two groups were homogeneous (Table 1).
There was a significant difference in the mean score of sleep quality in the group

Table 2 Comparison of mean scores of sleep quality in the study groups before and after intervention

\begin{tabular}{lccc}
\hline Sleep quality & Before intervention & After intervention & Paired $\mathrm{t}$ - test \\
\cline { 2 - 3 } Essential oil & Mean $\pm \mathrm{SD}^{*}$ & Mean $\pm \mathrm{SD}$ & \\
Placebo & $11.12 \pm 2.7$ & $22.2 \pm 9.60$ & $\begin{array}{c}\mathrm{p}=0.001 \\
\mathrm{t}=5.48, \mathrm{df}=49)\end{array}$ \\
Independent t- test & $11.14 \pm 2.6$ & $11.24 \pm 2.6$ & $\begin{array}{c}\mathrm{p}=0.09 \\
(\mathrm{t}=-0.742, \mathrm{df}=49)\end{array}$ \\
$\mathrm{p}=0.971$ & $\mathrm{p}=0.001$ & ---- \\
\hline
\end{tabular}

receiving Lavender essential oil before and after intervention (Table 2).

At the end of the intervention, a positive effect was observed on sleep quality in $64 \%$ of the patients in the group receiving Lavender essential oil. There was also a negative effect on sleep quality scores in $14 \%$ of the patients while $22 \%$ of the patients showed no certain changes in their quality of sleep. In the control group, $56 \%$ of the participants experienced no change in sleep quality than before, but there was a negative impact in $24 \%$ of the patients

Table 3 Comparison of sleep quality frequency and mean score in the study groups

\begin{tabular}{lccc}
\hline 7 Nights after intervention & $\begin{array}{c}\text { Lavender essential oil } \\
\text { (frequency and mean) }\end{array}$ & $\begin{array}{c}\text { Placebo } \\
\text { (frequency and mean) }\end{array}$ & p-value \\
\hline Positive effect & $32(64)$ & $10(20)$ & \\
Negative effect & $7(14)$ & $12(24)$ & $0.001^{*}$ \\
No change & $11(22)$ & $28(56)$ & \\
Total & $50(100)$ & $50(100)$ & \\
\hline
\end{tabular}

*Chi-squared test

and a positive effect in $20 \%$ of the patients in terms of sleep quality (Table 3 ).

\section{Discussion}

The results of the present study showed that there was a significant difference in the mean sleep quality between the intervention group receiving aromatherapy with lavender essential oil and the group treated with distilled water. Similar to this finding, the results of a pilot study conducted by Lewith et al. on ten volunteers showed that aromatherapy with lavender essential oil in the intervention group improved the mean sleep quality scores on the PSQI questionnaire. While, no significant changes were observed in the control group using sweet almond essential oil as a placebo [12]. Chien et al. [19] also came to similar results. Moeini et al. studied the effects of lavender essential oil on the sleep quality of patients in the Coronary Care Unit (CCU) and the results demonstrated a statistically significant difference in sleep quality between the control and case groups after aromatherapy with lavender essential oil [18]. In a pilot study, aromatherapy with lavender essential oil increased the time of night sleep and stopped or discontinued taking sedative drugs in two older adult patients with dementia during the night. In a study on three older adult patients with chronic insomnia, inhalation of lavender essential oil showed to be a good alternative to previous sedative drugs (sleeping pills) and patients reported better sleep quality compared to their quality of sleep when they were taking previous medications. Meanwhile no adverse effects were reported [20]. In line with this finding, in a controlled clinical trial on older adult patients with acute medical problems, $72 \%$ of patients who received aromatherapy with lavender essential reported a good quality of sleep. Furthermore, $79 \%$ of these patients reported that they had a good day after receiving aromatherapy at night. 
The value was $26 \%$ in the control group [21]. It is believed that lavender essential oil exerts its psychological effects through the limbic system, particularly through amygdala and hippocampus. Although mechanism of the effect of this plant is unclear at cellular level, several studies suggest that lavender has probably a function similar to benzodiazepines and causes an increase in GABA in the amygdala. After conducting a study, Re et al. concluded that linalool in lavender causes the release of acetylcholine and ionic performance changes in the neuromuscular junction point [22]. Linalyl acetate has narcotics function and linalool acts as a sedative drug. This function explains the sedative properties of lavender [22]. Nowadays aromatherapy is considered as a holistic nursing care and the use of complementary therapies such as aromatherapy is a part of professional activities of nurses [23]. On the other hand, complementary therapies, in addition to being cost-effective, in most cases have no serious adverse effects and drug interactions; and they are well accepted by patients [24]. Studies show that adverse effects of aromatherapy with lavender essential oil are very limited. Only two cases of headache and dizziness have been reported [25]. Consistent with the results of most studies, in the present study no special adverse effect was observed. Given the undeniable effect of environmental conditions on the quality of sleep and with regard to the fact that mental state of a person is effective on answering the questions, it is recommended that in future research polysomnography (a type of sleep study, is a multi-parametric test used in the study of sleep and as a diagnostic tool in sleep medicine) be used as a gold standard in the sleeprelated disorders to confirm the effectiveness of aromatherapy with lavender essential oil in improving sleep quality of older adults.

As the aging population is rising throughout the world, sleep quality improve in this age group should be considered further. However, further studies are needed to clarify the effects of aromatherapy on sleep disturbances of older adults.

\section{Conclusion}

Aromatherapy whit lavender essential oil as a non-pharmacological intervention improved sleep quality in older adults.

\section{Acknowledgements}

This paper has been adapted from a Master Thesis in geriatric nursing and it was granted and approved by Kashan University of Medical Sciences. Therefore, the authors would like to acknowledge Kashan University of Medical Science for supporting this research. We also would like to thank all of the older adults contributing in this study.

\section{Authors' contributions}

Study design: DA, FI

Data collection and analysis: DA, HA, NMA

Manuscript preparation: FI, NMA

All authors have read and approved the final version.

\section{Conflicts of Interest}

"The authors declare that they have no competing interests."

\section{Funding}

The author (s) received no financial support for the research, authorship, and/or publication of this article.

\section{Availability of data and materials}

The datasets used and/or analyzed during this study are available from the corresponding author on reasonable request.

\section{References}

1- Zisberg A, Gur-Yaish N, Shochat T. Contribution of routine to sleep quality in community elderly. Sleep 2010 ; 33(4): 509-14.

2- Tardy M, Gonthier R, Barthelemy JC, Roche F, Crawford-Achour E. Subjective sleep and cognitive complaints in 65 year old subjects: a significant association. The PROOF cohort. J Nutr Health Aging2015; 19(4): 424-30.

3- Sharif F, Seddigh M, Jahanbin I, Keshavarzi S. The effect of aerobic exercise on quantity and quality of sleep among elderly people referring to health centers of lar city, southern of Iran; A randomized controlled clinical trial. Curr Aging Sci2015; 8(3): 248-55.

4- De Almondes KM, Costa MV, Malloy-Diniz LF, Diniz BS. Insomnia and risk of dementia in older adults: Systematic review and meta-analysis. $J$ Psychiatr 
Res2016; 77: 109-15.

5- Endeshaw Y, Rice TB, Schwartz AV, et al. Snoring, daytime sleepiness, and incident cardiovascular disease in the health, aging, and body composition study. Sleep2013; 36(11): 1737-45.

6- Reid KJ, Baron KG, Lu B, Naylor E, Wolfe L, Zee PC. Aerobic exercise improves self-reported sleep and quality of life in older adults with insomnia. Sleep Med2010; 11(9): 934-40.

7- Campana LM, Clifford GD, Trinder J, Pittman SD, Malhotra A. A possible method to predict response to nonpharmacological insomnia therapy. J Clin Sleep Med2011; 7(4): $370-5$.

8- Karadag E, Samancioglu S, Ozden D, Bakir E. Effects of aromatherapy on sleep quality and anxiety of patients. Nurs Crit Care2017; 22(2): 105-12.

9- Burns A, Perry E, Holmes C, et al. A double-blind placebo-controlled randomized trial of Melissa officinalis oil and donepezil for the treatment of agitation in Alzheimer's disease. Dement Geriatr Cogn Disord2011; 31(2): 158-64.

10- Huang L, Abuhamdah S, Howes MJ, et al. Pharmacological profile of essential oils derived from Lavandula angustifolia and Melissa officinalis with antiagitation properties: focus on ligand-gated channels. $J$ Pharm Pharmacol2008; 60(11): 1515-22.

11- Goel N, Kim H, Lao RP. An olfactory stimulus modifies nighttime sleep in young men and women. Chronobiol Int2005; 22(5): 889-904.

12- Lewith GT, Godfrey AD, Prescott P. A single-blinded, randomized pilot study evaluating the aroma of Lavandula augustifolia as a treatment for mild insomnia. J Altern Complement Med2005; 11(4): 631-7.

13- Kasper S. An orally administered lavandula oil preparation (Silexan) for anxiety disorder and related conditions: an evidence based review. Int J Psychiatry Clin Pract2013; 17(1): 15-22.

14- Vitinius F, Hellmich M, Matthies A, et al. Feasibility of an interval, inspiration-triggered nocturnal odorant application by a novel device: a patient-blinded, randomised crossover, pilot trial on mood and sleep quality of depressed female inpatients. Eur Arch Otorhinolaryngol2014; 271(9): 2443-54.
15- Budur C, Rodriguez C, Foldvary-Schaefer N. Advances in treating insomnia. Cleve Clin J Med2007; 74(4): 251-2, 255-8, 261-2 passim.

16- Buysse DJ, Reynolds CF, Monk TH, Berman SR, Kupfer DJ. The pittsburgh sleep quality index: a new instrument for psychiatric practice and research. Psychiatry Res1989; 28(2): 193-213.

17- Adib-Hajbaghery M, Izadi-Avanji F, Akbari H. Quality of sleep and its related risk factors in hospitalized older patients in Kashan's Hospitals, Iran 2009. Iran $J$ Nurs Midwifery Res2012; 17(6): 414-20.

18- Moeini M, Khadibi M, Bekhradi R, Mahmoudian SA, Nazari F. Effect of aromatherapy on the quality of sleep in ischemic heart disease patients hospitalized in intensive care units of heart hospitals of the Isfahan university of medical sciences. Iran J Nurs Midwifery Res2010; 15(4): 234-9.

19- Chien LW, Cheng SL, Liu CF. The effect of lavender aromatherapy on autonomic nervous system in midlife women with insomnia. Evid Based Complement Alternat Med2012; 2012: 740813.

20- Altinyazar V, Kiylioglu N. Insomnia and dementia: is agomelatine treatment helpful? Case report and review of the literature. Ther Adv Psychopharmacol2016; 6(4): 263-8.

21- Lin PW, Chan WC, Ng BF, Lam LC. Efficacy of aromatherapy (Lavandula angustifolia) as an intervention for agitated behaviours in Chinese older persons with dementia: a cross-over randomized trial. Int $J$ Geriatr Psychiatry2007; 22(5): 405-10.

22- Re L, Barocci S, Sonnino S, et al. Linalool modifies the nicotinic receptor-ion channel kinetics at the mouse neuromuscular junction. Pharmacol Res2000; 42(2): 177-82.

23- Frisch NC. Standards for holistic nursing practice: a way to think about our care that includes complementary and alternative modalities. Online J Issues Nurs2001; $6(2): 4$.

24- Cooke M, Rapchuk I, Doi SA, et al. Wrist acupressure for post-operative nausea and vomiting (WrAP): A pilot study. Complement Ther Med2015; 23(3): 372-80.

25- Barre L. Aromatherapy in nursing homes. Soins 2015 ; (797): 10-2.

Copyright(C 2016 ASP Ins. This open-access article is published under the terms of the Creative Commons Attribution-NonCommercial 4.0 International License which permits Share (copy and redistribute the material in any medium or format) and Adapt (remix, transform, and build upon the material) under the Attribution-NonCommercial terms. 